\title{
Calcium/Calmodulin-dependent Protein Kinase II (CaMKII) Signaling in Cardiomyocytes Initiates Inflammatory Gene Expression, Inflammasome Activation and Macrophage Accumulation Leading to Adverse Cardiac Remodeling in Response to Pressure Overload
}

\author{
Takeshi Suetomi, Andrew Willeford, Michelle Li, Shigeki Miyamoto, Joan Heller Brown
}

Pharmacology, University of California San Diego, USA

Background: Inflammation is associated with cardiac remodeling and heart failure, but how it is initiated in response to non-ischemic interventions is not known. We tested the hypothesis that the activation of CaMKII in cardiomyocytes stimulates inflammatory gene expression and subsequent responses that contribute to adverse remodeling following transverse aortic constriction (TAC).

Methods and Results: Mice in which CaMKIIdelta was selectively deleted from cardiomyocytes (Cardiac specific knockout; $\mathrm{CKO}$ ) and $\mathrm{fl} / \mathrm{fl}$ control (CTL) mice were subjected to pressure overload by TAC. Significant increases in CaMKII activity and NFkB activation were observed in CTL but not in CKO hearts at 1.5 days of TAC. Cardiac mRNA levels for pro-inflammatory chemokines and cytokines increased by 1.5 days and peaked at 3 days with 5-20 fold increases vs sham in MCP-1, MIP1alpha, and IL-6. These responses were decreased by 40-55\% in the CKO mice. Cardiomyocytes isolated from CTL and CKO mice following TAC showed similar increases in gene expression and attenuation when CaMKII was deleted. Priming and activation of inflammasomes was assessed by measuring NLRP3 mRNA levels and caspase1 activity at 3 days of TAC. Both were significantly increased in CTL but not in CKO hearts and isolated cardiomyocytes. Immunohistochemical analysis revealed significant CD68+ macrophage accumulation by 7 and 14 days of TAC which was significantly attenuated in the CKO mice. Fibrosis assessed by Masson-trichrome staining, collagen (col1a1, col3a1) and periostin mRNA expressions occurred slightly later and was clearly elevated at 14-28 days TAC and also significantly attenuated in the CKO mice. Pharmacologic NLRP3 blockade between days 1-7 after TAC significantly reduced of CD68 positive cell accumulation at 14days of TAC and fibrosis at 28 days of TAC.

Conclusions: Activation of CaMKII localized in cardiomyocytes leads to NFkB signaling and initiates inflammatory transcriptional programs and inflammasome activation within cardiomyocytes. The functional significance of this response in driving subsequent immune cell recruitment, fibrosis and adverse remodeling is suggested by our studies and is being further investigated through pharmacologic and genetic blockade. Our work may establish the critical importance of early inflammatory responses to cardiomyocyte CaMKII signaling as targets to prevent progression from hypertrophy to heart failure. 